Transport and Communications Science Journal, Vol. 71, Issue 4 (05/2020), 419-430

Transport and Communications Science Journal

\title{
MODELING OF CONTACT INTERFACE BETWEEN TWO MATERIAL LAYERS IN HYBRID STRUCTURES
}

\author{
Nguyen Thi Thu Nga ${ }^{1 *}$, Tran Nam Hung \\ Le Quy Don Technical University, No. 236 Hoang Quoc Viet Street, Hanoi, Vietnam.
}

ARTICLE INFO

TYPE: Research Article

Received: $10 / 4 / 2020$

Revised: $17 / 5 / 2020$

Accepted: 18/5/2020

Published online: $28 / 5 / 2020$

https://doi.org/10.25073/tcsj.71.4.10

* Corresponding author

Email: nguyennga@lqdtu.edu.vn

\begin{abstract}
In hybrid structures, material layers of different mechanical properties are integrated to increase bearing capacity. When the difference in mechanical properties or thickness of the material layers is very large, debonding usually occurs along the interface between the two layers. This study uses a homogenization procedure combined with asymptotic algorithm applied on weaker/thinner materials to determine the interface stiffnesses for such structures. All the material layers and the interface are assumed to be linear elastic. Comprising with the available methods and numerical simulation results showed that the proposed model is more suitable with the work of the structures in reality. Furthermore, in this method the interface stiffnesses can be easily determined through the number and length of cracks and the dry or saturated state of the medium are also considered.
\end{abstract}

Keywords: Hybrid structures, interface stiffnesses, homogenization, asymptotic algorithm

(C) 2020 University of Transport and Communications

\section{INTRODUCTION}

Hybrid structures are made of structures and layers with different mechanical properties to significantly improve the structural strength. Nowadays, they are being studied and used, for example, a combination of textile-reinforced concrete containing fine-grained concrete and lightweight concrete [1], fiber reinforced cementitious matrix composite and concrete [2], 
Transport and Communications Science Journal, Vol. 71, Issue 4 (05/2020), 419-430

concrete beam and fiber reinforced polymers composite [3], flexible pavement and semi-rigid pavement in the transportation engineering, etc. Experimental studies showed that debonding can occur at the matrix-fiber interface or at the matrix-concrete interface due to differences between materials, such as rigidity, crack density, porous density. To simulate this type of structures, there are two micromechanical models. The first one is discrete model, which can show interconnected classes by the irregular Signorini [4], regular Newton-Euler [5] or Coulomb's law [6]. These methods require lot of parameters in numerical simulations. This is not effective for large structural simulations. The second one is a continuous model assuming that there is a new layer between the two material layers so-called the interface layer, which has zero thickness and characterized by normal and tangential stiffnesses $C_{N}, C_{T}$ :

$$
C_{N}=\lim _{e \rightarrow 0} \frac{\bar{L}_{3333}}{e}, \quad C_{T}=\lim _{e \rightarrow 0} \frac{\bar{L}_{1313}}{e}
$$

where $\bar{L}_{3333}, \bar{L}_{1313}$ are the components of the effective stiffness tensor $\overline{\mathbb{L}}$. The effective compliance tensor $\overline{\mathbb{S}}=\overline{\mathbb{L}}^{-1}$ is written in the form:

$$
\overline{\mathbb{S}}=\left(\begin{array}{cccccc}
\frac{1}{\bar{E}_{1}} & -\frac{\bar{v}_{12}}{\bar{E}_{1}} & -\frac{\bar{v}_{13}}{\bar{E}_{1}} & 0 & 0 & 0 \\
-\frac{\bar{v}_{21}}{\bar{E}_{2}} & \frac{1}{\bar{E}_{2}} & -\frac{\bar{v}_{23}}{\bar{E}_{2}} & 0 & 0 & 0 \\
-\frac{\bar{v}_{31}}{\bar{E}_{3}} & -\frac{\bar{v}_{32}}{\bar{E}_{3}} & \frac{1}{\bar{E}_{3}} & 0 & 0 & 0 \\
0 & 0 & 0 & \frac{1}{\bar{\mu}_{23}} & 0 & 0 \\
0 & 0 & 0 & 0 & \frac{1}{\bar{\mu}_{31}} & 0 \\
0 & 0 & 0 & 0 & 0 & \frac{1}{\bar{\mu}_{12}}
\end{array}\right)
$$

where $\bar{E}_{i}$ are effective Young's moduli, $\bar{\mu}_{i j}$ are effective shear moduli and $\bar{v}_{i j}$ are effective Poisson's ratios.

Rekik et al. [7] proposed a methodology for determining $C_{N}, C_{T}$ of the damaging interface that includes the coupling between the homogenization theory and the asymptotic techniques. This procedure requires three steps illustrated in Fig. 1. In this work, the interface appears as a third material between brick and mortar and is made of a mixture of brick and mortar by the exact analytical homogenization of a laminate of the two layers. This closed-form solution is validated in the condition of volume fractions of phases and material properties: $\varphi_{b} \approx \varphi_{m}, E_{m} \approx E_{b}$. 
Transport and Communications Science Journal, Vol. 71, Issue 4 (05/2020), 419-430

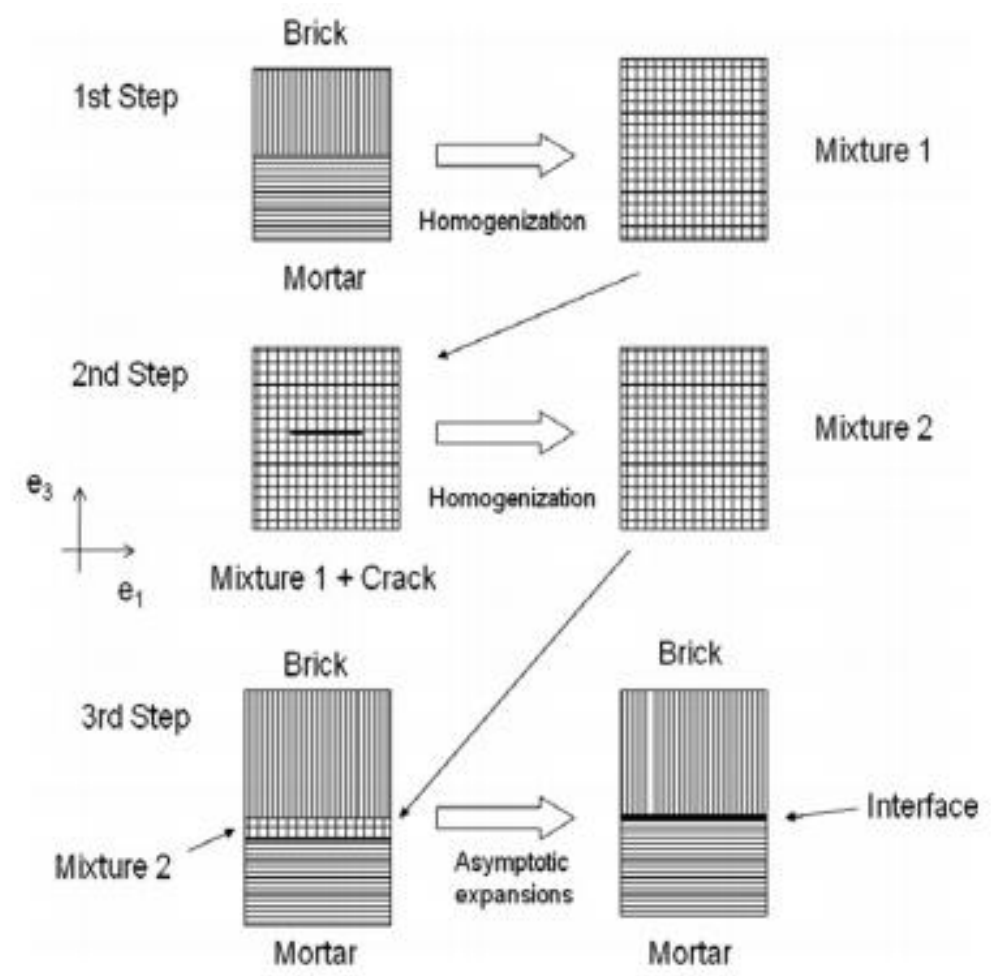

Figure 1. Principle of brick and mortar's interface [7].

The normal and tangential joint stiffnesses are finally obtained by the coupling between homogenization technique and asymptotic analysis:

$$
C_{N}^{\mathrm{Re}}=\frac{S_{0}}{2 C(1+D) a^{3}} ; C_{T}^{\mathrm{Re}}=\frac{S_{0}}{4 C(1-D) a^{3}}
$$

where $a$ is crack half-length; $S_{0}$ denotes the joint area in 3D applications; C, D are two parameters which depends on the effective elastic engineering constants of the crack-free material (HEM-1) as follows:

$$
\left\{\begin{array}{l}
C=\frac{\pi}{4} \frac{\sqrt{E_{1}^{0}}+\sqrt{E_{3}^{0}}}{\sqrt{E_{1}^{0} E_{3}^{0}}}\left(\frac{1}{G_{13}^{0}}-2 \frac{v_{13}^{0}}{E_{1}^{0}}+\frac{2}{\sqrt{E_{1}^{0} E_{3}^{0}}}\right)^{\frac{1}{2}} \\
D=\frac{\sqrt{E_{1}^{0}}-\sqrt{E_{3}^{0}}}{\sqrt{E_{1}^{0}}+\sqrt{E_{3}^{0}}}
\end{array}\right.
$$

For more detail, the readers can see in [7].

However, in the hybrid structures the layers are usually much different in thickness and material properties. Besides, the debonding normally occurs in the interface and depends strongly on the weaker layer [2, 3, 8]. Therefore, the properties of this third material must be obtained by performing an exact linear homogenization procedure on the weaker layer, and then $C_{N}, C_{T}$ are determined by applying an asymptotic limit analysis procedure to the equivalent homogeneous material. The proposed procedure is shown in Fig. 2 with only two steps. 
Transport and Communications Science Journal, Vol. 71, Issue 4 (05/2020), 419-430

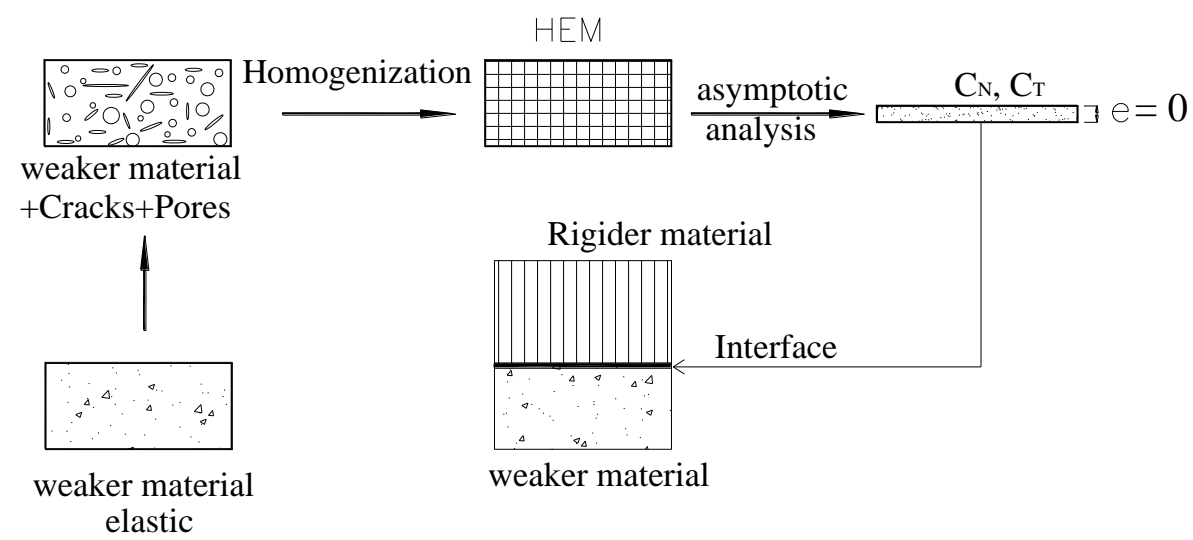

Figure 2. Proposed multi-scale interface methodology.

In this work, we suppose that only the weaker material is considered to determine the law of interface. Two steps are conducted. Firstly, the effective properties of the weaker material with cracks and pores are characterized by using homogenization techniques, so-called homogeneous equivalent medium (HEM). Secondly, using asymptotic techniques, the thickness of this material tends to zero in order to model HEM as an interface.

\section{SCOPE OF THE STUDY}

It is well known that the weaker layer normally has micro-cracks and pores. For the sake of simplicity, only parallel orientation distribution of cracks will be considered. This leads to anisotropy of the overall response of effective homogenized material. In this study the layers are supposed to be isotropic and linear elastic materials where the rigider layer is safe (uncracked) and the weaker one is saturated/non-saturated micro-cracked. The homogenization of the micro-cracked material can be carried out exactly using an analytical homogenization formulation as described in [9] for non-saturated case and in [10, 11, 12] for saturated case. Then, the asymptotic limit analysis is performed to define the expression of the normal and tangential stiffnesses. The crack density is determined as below:

$$
d_{c}=\frac{n_{c} a^{3}}{\delta S}
$$

where $n_{c}$ is number of cracks per unit volume, $\mathrm{S}$ is surface area, $\delta$ denotes thickness of the weaker material.

The influence of the crack density on the interface law will be discussed in the fifth part of the present paper.

\section{INTERFACE LAW FOR DRY MEDIUM}

The Mori-Tanaka scheme allows taking into account the interactions between cracks even if the crack density is low. According to the well-known result of Eshelby (1957), this is achieved quite easily since the strain field is homogeneous within an ellipsoidal inclusion embedded in an infinite medium subjected to the constant strain at infinity: $\mathbb{E}^{0}=\langle\varepsilon\rangle^{m}$ (see in [9]). The overall average strain $\langle\varepsilon\rangle$ is defined through the relationship: 
Transport and Communications Science Journal, Vol. 71, Issue 4 (05/2020), 419-430

$\mathbb{E}=\langle\varepsilon\rangle=(1-\varphi)\langle\varepsilon\rangle^{m}+\varphi\langle\varepsilon\rangle^{c}=(1-\varphi) \mathbb{E}^{0}+\varphi \overline{\mathbb{A}}^{c} \mathbb{E}^{0}$ where $\langle\varepsilon\rangle^{m},\langle\varepsilon\rangle^{c}$ are respectively average strain in the matrix and in the cracks, $\varphi$ denotes the volume fraction, $\overline{\mathbb{A}}^{c}$ is the fourth-order localization tensor and related to $\langle\varepsilon\rangle^{c}$ and $\mathbb{E}^{0}$ by: $\langle\varepsilon\rangle^{c}=\overline{\mathbb{A}}^{c} \mathbb{E}^{0}$.

In [13], a closed-form predictions for $\overline{\mathbb{A}}^{M T}$ is provided, respect to the boundary condition $\mathbb{E}^{0}=\overline{\mathbb{A}}^{M T} \mathbb{E}$, as $\overline{\mathbb{A}}^{M T}=\overline{\mathbb{A}}^{m}=\left[(1-\varphi) \mathbb{I}+\varphi \overline{\mathbb{A}}^{c}\right]^{-1}$. Therefore, the effective stiffness tensor is defined as: $\overline{\mathbb{L}}^{M T}=(1-\varphi) \mathbb{L}^{m}: \mathbb{H}$ with $\mathbb{H}=\left[(1-\varphi) \mathbb{I}+\varphi \overline{\mathbb{A}}^{c}\right]^{-1}$. If the parallel cracks are considered in the initially isotropic material, the effective material is transversely isotropic. In this case, $\varphi=\frac{4 \pi}{3} d_{c} X$ with $X \rightarrow 0$ and the effective stiffness tensor takes the form in the Walpole coordinates as follows:

$$
\overline{\mathbb{L}}^{M T}=\sum_{1}^{6} c_{i}^{M T} \mathbb{E}_{i}
$$

in which

$$
\begin{aligned}
& c_{1}^{M T}=\frac{2 \mu^{m}\left[3+16 d_{c}\left(1-v^{m 2}\right)\right]}{16 d_{c}\left(1-v^{m}\right)^{2}+3\left(1-2 v^{m}\right)}, \quad c_{2}^{M T}=\frac{6 \mu^{m}\left(1-v^{m s}\right)}{16 d_{c}\left(1-v^{m}\right)^{2}+3\left(1-2 v^{m}\right)} c_{3}^{M T}=2 \mu^{m}, \\
& c_{4}^{M T}=\frac{6 \mu^{m}\left(1-2 v^{m}\right)}{16 d_{c}\left(1-v^{m}\right)-3\left(1-2 v^{m}\right)}, c_{5}^{M T}=c_{6}^{M T}=\frac{6 \mu^{m} v^{m}}{16 d_{c}\left(1-v^{m}\right)^{2}+3\left(1-2 v^{m}\right)}=\frac{v^{m}}{\left(1-v^{m}\right)} c_{2}^{M T}
\end{aligned}
$$

and $\mu^{m}, v^{m}$ being respectively shear modulus and Poisson's ratio of the matrix.

Inverting the stiffness tensor $\overline{\mathbb{L}}^{M T}$ gives the corresponding compliance tensor $\overline{\mathbb{S}}^{M T}$ associated with the properties of HEM (see Fig. 1 and Eq. (2)). The expressions for the interface stiffness $C_{N}, C_{T}$ in Eq. (1) read:

$$
C_{N}^{c \backslash \backslash M T}=\frac{1}{d_{c}} \frac{3 E^{m}}{16 \delta\left(1-v^{m^{2}}\right)} ; C_{T}^{c \backslash M M T}=\frac{1}{d_{c}} \frac{3 \mu^{m}}{16 \delta\left(1-v^{m}\right)}
$$

Eq. (7) implies that $C_{N}^{c \backslash M M T}=2 C_{T}^{c \backslash \backslash M T}$.

\section{INTERFACE LAW FOR SATURATED MEDIUM}

\subsection{Thomsen's model}

Thomsen proposed the effective elastic behavior of elastic isotropic medium containing saturated micro-cracks with parallel distribution [10]. This theory developed under the conditions of balanced pressures, non-interaction between cracks and non-rupture. Recall that the effective compliance tensor is given in Eq. (2) with the following components: 
Transport and Communications Science Journal, Vol. 71, Issue 4 (05/2020), 419-430

$$
\left\{\begin{array}{l}
\frac{1}{\bar{E}_{1}}=\frac{1}{\bar{E}_{2}}=\frac{1}{E^{m}}-\frac{\frac{1}{9}\left(\frac{\phi_{p}}{k_{\phi_{p}}}\right)^{2}}{\phi_{t} C_{t}}, \quad-\frac{\bar{v}_{12}}{\bar{E}_{1}}=-\frac{\bar{v}_{13}}{\bar{E}_{1}}=-\frac{v^{m}}{E^{m}}-\frac{\frac{1}{9}\left(\frac{\phi_{p}}{k_{\phi_{p}}}\right)^{2}}{\phi_{t} C_{t}} ; \frac{1}{\bar{E}_{3}}=\frac{1}{\bar{E}_{1}}+Z_{N}^{m} ; \\
\frac{1}{\bar{\mu}_{23}}=\frac{1}{\bar{\mu}_{31}}=\frac{1}{\mu^{m}}+Z_{T}^{m} ; \frac{1}{\bar{\mu}_{12}}=\frac{1}{\mu^{m}} ; Z_{N}^{s}=\frac{16}{3} \frac{1}{E^{m}}\left(1-v^{m}\right)\left(1+v^{m}\right) d_{c}\left(1-\frac{k_{f}}{k_{0}}\right) D_{c p} \\
Z_{T}^{s}=\frac{16}{3} \frac{1}{\mu_{d}} \frac{1-v^{m}}{2-v^{m}} d_{c}, \quad D_{c p}=\frac{1}{1-\frac{k_{f}}{k_{0}}+\frac{k_{f}}{\phi_{p}+\phi_{c}}\left(\frac{1}{k_{m}}-\frac{1}{k_{0}}+Z_{N}^{d}\right)} ; \\
\phi_{c}=d_{c} \gamma \frac{4 \pi}{3}, \quad \phi_{p}=\frac{N_{p} V_{p}}{V}=N_{p} \frac{4 \pi b^{3}}{3 e . S}, \frac{1}{k_{\phi_{p}}}=\frac{1}{\phi_{p}}\left(\frac{1}{k_{m}}-\frac{1}{k_{0}}\right) ; \\
\phi_{t} C_{t}=\phi_{p} C_{p}+\phi_{c} C_{c} ; \phi_{p} C_{p}=\left(\frac{1}{k_{m}}-\frac{1}{k_{0}}\right)+\left(\frac{1}{k_{f}}-\frac{1}{k_{0}}\right) \phi_{p}, \quad \phi_{c} C_{c}=Z_{N}^{d}+\left(\frac{1}{k_{f}}-\frac{1}{k_{0}}\right) \phi_{c}
\end{array}\right.
$$

where $k_{m}, k_{f}, k_{0}$ are respectively the compressibility moduli of the dry matrix, saturated fluid and uncracked matrix. According to the asymptotic analysis in Eq. (1), the expressions for the interface stiffnesses $C_{N}$ and $C_{T}$ read:

$$
C_{N}^{c \backslash}=\frac{1}{d_{c}} \frac{3}{16 \delta}\left(\frac{4 k_{0} k_{f}}{\gamma \pi\left(k_{0}-k_{f}\right)}+\frac{E^{m}}{\left(1-v^{m}\right)\left(1+v^{m}\right)}\right) ; C_{T}^{c \|}=\frac{1}{d_{c}} \frac{3 \mu_{0}\left(2-v^{m}\right)}{16 \delta\left(1-v^{m}\right)}
$$

with $\gamma=a_{3} / a<1$ being aspect ratio of the cracks.

\subsection{B\&K's model}

Considering an incremental external pressure, Brown and Korringa (B\&K) made no assumptions about the shape of the crack [11]. Both B\&K's and Thomsen's models give the same expression of the corresponding compliance tensor, but B\&K's model uses the parameters

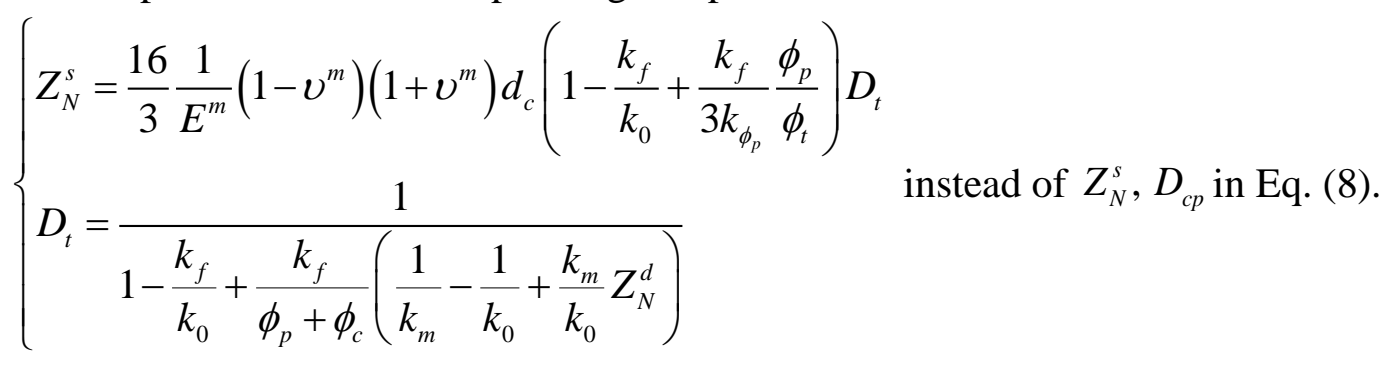

The interface stiffnesses $C_{N}, C_{T}$ obtained in Eq. (10) have the same expressions with the ones of Thomsen's model if $k_{0}=k^{m}$, i.e., the uncracked matrix is dry.

$$
C_{N}^{c \backslash}=\frac{1}{d_{c}} \frac{3}{16 \delta}\left(\frac{4 k^{m} k_{f}}{\gamma \pi\left(k_{0}-k_{f}\right)}+\frac{E^{m}}{\left(1-v^{m}\right)\left(1+v^{m}\right)}\right) ; C_{T}^{c \backslash \backslash}=\frac{1}{d_{c}} \frac{3 \mu^{m}\left(2-v^{m}\right)}{16 \delta\left(1-v^{m}\right)}
$$




\subsection{S\&K's model}

Shafiro and Kachanov (S\&K) [12] consider linear elastic solid containing cracks of diverse shapes and arbitrary orientation distributions. Two types of shape considered in this study are needle-shaped spheroidal and crack-like spheroidal cavities filled with non-viscous compressible fluid, which is characterized by the fluid compressibility $\kappa$. This solution takes into account the stress interactions between the cracks in analyzing in terms of elastic potentials.

In case of parallel cracks, the components of Eq. (2) can be defined as:

$$
\begin{aligned}
& \bar{E}_{1}=\bar{E}_{2}=E_{0}, \bar{\mu}_{31}=\bar{\mu}_{23}, \bar{v}_{12}=\bar{v}_{21}=v_{0}, \bar{v}_{13}=\bar{v}_{23}=\frac{\bar{E}_{1}}{\bar{E}_{3}} \bar{v}_{31} \text { and } \\
& \frac{E_{0}}{\bar{E}_{3}}=1+\frac{16}{3}\left(1-v_{0}^{2}\right)\left(\rho-\rho_{1}\right) ; \frac{\mu_{0}}{\bar{\mu}_{31}}=1+\rho \frac{16}{3} \frac{\left(1-v_{0}\right)}{\left(2-v_{0}\right)}, \bar{\mu}_{12}=\frac{E_{1}}{2\left(1+v_{0}\right)}, \bar{v}_{31}=\frac{3 v_{0}}{3+16\left(1-v_{0}^{2}\right)\left(\rho-\rho_{1}\right)}
\end{aligned}
$$

where $\rho=\frac{1}{V} \sum\left(a^{3}\right)^{(k)}=d_{c}, \quad \rho_{1}=\frac{1}{V} \sum\left(\frac{a^{3}}{1+\delta_{c}}\right)^{(k)}, \delta_{c}=\gamma \frac{\pi}{4\left(1-v_{0}^{2}\right)}\left[\kappa E_{0}-3\left(1-2 v_{0}\right)\right]$.

The normal and tangential interface stiffnesses are given by:

$$
C_{N}^{c \backslash}=\frac{1}{d_{c}} \frac{3}{16 \delta}\left(\frac{4 k_{0}}{\gamma \pi\left(k_{0} \kappa-1\right)}+\frac{E_{0}}{\left(1-v_{0}^{2}\right)}\right), \quad C_{T}^{c \backslash \backslash}=\frac{1}{d_{c}} \frac{3 \mu_{0}\left(2-v_{0}\right)}{16 \delta\left(1-v_{0}\right)}
$$

\section{DISCUSSION}

The expresstions of $C_{N}, C_{T}$ in Eqs. (7), (9), (10) and (11) show that $C_{N}, C_{T}$ depend not only on the properties and thickness of the weaker material, but also strongly on the crack density $d_{c}$. Besides, in case of dry matrix ( $k_{f}=0$ ), the expression of $C_{N}^{c l}$ in Eq. (9) becomes exactly the one of Mori-Tanaka's expression (see Eq. (7)). Eq. (11) indicates that if the fluid compressibility is the inverse of the fluid compressibility modulus $\left(\kappa=1 / k_{f}\right)$ and the initial crack density is negligible $\left(k^{m} \approx k_{0}, E^{m} \approx E_{0}, \mu^{m} \approx \mu_{0}\right)$, the expressions of $C_{N}, C_{T}$ of Thomsen's model in Eq. (9), B\&K's model in Eq. (10) and S\&K's model in Eq. (11) are very close.

In comparing with Rekik's expression in dry case (Eq. (3)), the expression of MoriTanaka (Eq. (7)) is simpler. If one supposes that the two materials have the same properties $\left(E_{0}, v\right)$, Eq. (3) leads to $C=\frac{\pi}{E_{0}}, \mathrm{D}=0$, and therefore $C_{N}^{\mathrm{Re}}=\frac{1}{2 \pi} \frac{E_{0} S_{0}}{a_{r k}^{3}}, C_{T}^{\mathrm{Re}}=\frac{1}{4 \pi} \frac{E_{0} S_{0}}{a_{r k}^{3}}$ while Eq. (7) gives $C_{N}^{M T}=\frac{3}{16\left(1-v^{2}\right)} \frac{E_{0} S_{0}}{n_{c} a_{M T}^{3}}, C_{T}^{M T}=\frac{3}{32\left(1-v^{2}\right)} \frac{E_{0} S_{0}}{n_{c} a_{M T}^{3}}$. It can be seen that if $a_{r k}=a_{M T}$, the ratios $\frac{C_{N}^{\mathrm{Re}}}{C_{N}^{M T}}=\frac{C_{T}^{\mathrm{Re}}}{C_{T}^{M T}} \approx(0.72 \div 0.85) n_{c}$ and all expressions have the same term $\frac{E_{0} S_{0}}{a^{3}}$. Note that the crack half-length $a_{r k}$ in Eq. (3) depends on the load that is calculated from experimental 'stress-displacement' diagrams obtained on the structure subjected to shear conditions whereas 
$a_{M T}$ in Eq. (7) is the average length of $n_{c}$ cracks in one material that can be easily observed and counted by using a special device. This is an advantage of the proposed model in this work.

Let us consider three cases of the properties of hybrid material (see Table 1) to discuss about the application capacity of the proposed model for the interface stiffnesses. The first one uses the topping material of ten times rigider than the base material while their thicknesses are the same. The second one considers only remarkable difference in the thicknesses and the last one refers remarkable differences in both rigidity and thickness of the two materials (see Fig. 3).

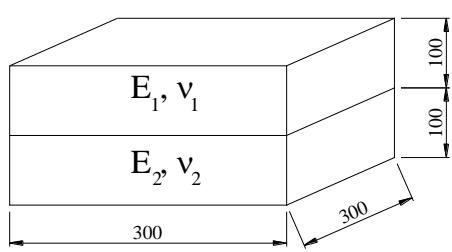

Case 1: $\mathrm{E}_{1}=10 \mathrm{E}_{2}, \mathrm{~h}_{1}=\mathrm{h}_{2}$

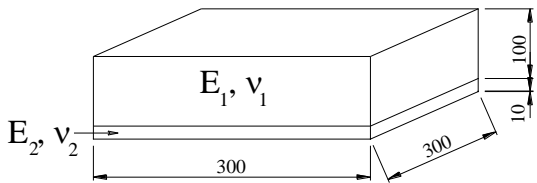

Case $2: \mathrm{h}_{1}=10 \mathrm{~h}_{2}, \mathrm{E}_{1}^{\sim} \mathrm{E}_{2}$

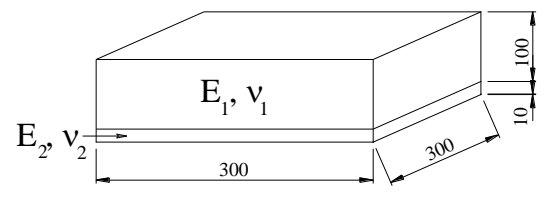

Case 3: $\mathrm{h}_{1}=10 \mathrm{~h}_{2}, \mathrm{E}_{1}=10 \mathrm{E}_{2}$

Figure 3. Dimensions and properties of the specimen for three cases of test.

Table 1. The properties of hybrid structure components.

\begin{tabular}{|c|c|c|c|c|c|c|c|c|}
\hline Cases & Materials & $\begin{array}{c}E_{0} \\
(M P a)\end{array}$ & $v_{0}$ & $\begin{array}{l}h \\
(\mathrm{~mm})\end{array}$ & $\begin{array}{c}C_{N}^{c \Downarrow l} \\
\text { Eq. (7) } \\
\left(\mathrm{N} / \mathrm{mm}^{3}\right)\end{array}$ & $\begin{array}{c}C_{T}^{c \backslash} \\
\text { Eq. (7) } \\
\left(\mathrm{N} / \mathrm{mm}^{3}\right)\end{array}$ & $\begin{array}{c}C_{N} \\
\text { Rekik } \\
\left(\mathrm{N} / \mathrm{mm}^{3}\right)\end{array}$ & $\begin{array}{c}C_{T} \\
\text { Rekik } \\
\left(\mathrm{N} / \mathrm{mm}^{3}\right)\end{array}$ \\
\hline \multirow{2}{*}{1} & $\begin{array}{l}\text { Topping } \\
\text { material }\end{array}$ & 50000 & 0.2 & 100 & $8.69 \times 10^{7}$ & $4.34 \times 10^{7}$ & $14.56 \times 10^{7}$ & $12.37 \times 10^{7}$ \\
\hline & $\begin{array}{c}\text { Base } \\
\text { material }\end{array}$ & 5000 & 0.17 & 100 & $n_{c} a_{M T}^{3}$ & $n_{c} a_{M T}^{3}$ & $a_{r k}^{3}$ & $a_{r k}^{3}$ \\
\hline \multirow{2}{*}{$2 a$} & $\begin{array}{l}\text { Topping } \\
\text { material }\end{array}$ & 9438 & 0.13 & 100 & $7.42 \times 10^{7}$ & $3.71 \times 10^{7}$ & $8.54 \times 10^{7}$ & $4.50 \times 10^{7}$ \\
\hline & $\begin{array}{c}\text { Base } \\
\text { material }\end{array}$ & 4000 & 0.3 & 10 & $n_{c} a_{M T}^{3}$ & $n_{c} a_{M T}^{3}$ & $a_{r k}^{3}$ & $a_{r k}^{3}$ \\
\hline \multirow[b]{2}{*}{$2 b$} & $\begin{array}{l}\text { Topping } \\
\text { material }\end{array}$ & 5000 & 0.17 & 100 & $8.69 \times 10^{7}$ & $4.34 \times 10^{7}$ & $7.16 \times 10^{7}$ & $3.58 \times 10^{7}$ \\
\hline & $\begin{array}{c}\text { Base } \\
\text { material }\end{array}$ & 5000 & 0.17 & 10 & $n_{c} a_{M T}^{3}$ & $n_{c} a_{M T}^{3}$ & $a_{r k}^{3}$ & $\overline{a_{r k}^{3}}$ \\
\hline \multirow{2}{*}{3} & $\begin{array}{l}\text { Topping } \\
\text { material }\end{array}$ & 50000 & 0.2 & 100 & $8.69 \times 10^{7}$ & $4.34 \times 10^{7}$ & $14.56 \times 10^{7}$ & $12.37 \times 10^{7}$ \\
\hline & $\begin{array}{c}\text { Base } \\
\text { material } \\
\end{array}$ & 5000 & 0.17 & 10 & $n_{c} a_{M T}^{3}$ & $n_{c} a_{M T}^{3}$ & $a_{r k}^{3}$ & $a_{r k}^{3}$ \\
\hline
\end{tabular}

Note that in Table 1, the normal and tangential stiffnesses $C_{N}, C_{T}$ of dry medium are derived using expressions (7) for proposed model and (3) for Rekik's model assuming the existence of an equal volume fraction between these two materials. Case 2 ( $2 \mathrm{a}$ and $2 \mathrm{~b}$ ) shows that the stiffnesses of proposed model and Rekik's model are in a good agreement in expression when there are small differences between material properties. Besides, it is observed that Rekik's model gives the same values of $C_{N}, C_{T}$ for cases 1 and 3. However, in the proposed model $C_{N}, C_{T}$ increase with respect to the decrease of phase height because of appearing of $n_{c}$ 
in the expressions of $C_{N}, C_{T}$. This is more appropriate in reality. Furthermore, the results of experimental test showed that debonding runs normally along the interface between the two layers $[2,7]$. Therefore, the proposed model of interface stiffnesses could be better than Rekik's to model a hybrid structure when the thickness and/or the stiffness of one material are much lower than those of the other (case 1 and 3). In addition, Rekik hasn't considered yet the case of parallel cracks full filled with compressible fluid that is taken into account in the proposed model. Consequently, the proposed method is also suitable to model the contact interface between two material layers in hybrid structure in the saturated state.

We study the behavior of a 3D model using Cast3M software. This is a finite element code for structural and fluid mechanics in which partial differential equations solved thanks to the finite element method. The user can propose developments to be integrated to the Cast $3 \mathrm{M}$ standard version. Cast $3 \mathrm{M}$ is a powerful software in simulating interface between two materials.

In order to validate the capacity of the proposed model, we consider the interface stiffnesses for case 1 . The dimension of the specimen is $300 \times 300 \times 100 \mathrm{~mm}$ and a "push - off" test is simulated. The three-dimensional interface elements used in Cast3M code is JOI4 and supposed to be elastic. This structure subjected to the force on one lateral surface of the top phase that is increased at a constant rate of $1 \mathrm{~N} / \mathrm{mm}^{2}$ (Fig. 4). Because the number of cracks $n_{c}$ is in the range of 1 to thousands, the crack length is initiated at certain $\mu m$, but under loading it may extend up to several $\mathrm{cm}$ [17]. Therefore, the value of interface stiffnesses varies significantly. It should be noted that if the interface stiffnesses are much greater than those of the basic material, the behavior of the structure with or without interface are the same, this means the layers are perfectly bonded.

To evaluate the influence of the stiffnesses on the interface shear failure, FE models will be applied with horizontal load that help to see relative sliding of the two layers. In fact, as sliding progresses the stress increases due to the presence of friction, the length of crack and/or number of crack increase. Therefore, $C_{N}, C_{T}$ decrease and depend on stress. For the sake of simplicity, we consider the behavior of the structure without propagation of cracks. Thus, the interface behavior in this state can be assumed to be elastic. Three tests are simulated: a perfect interface is used for the first test (case (a)); the second test considers $C_{N}, C_{T}$ at large values $\left(a=10^{-3} \mathrm{~mm}\right.$ and $n_{c}=100, C_{N_{-} M T}^{c \backslash \mid}=8.688 \times 10^{11}, C_{T_{-} M T}^{c \| 1}=4.344 \times 10^{11}$, case (b)) and the last one uses $C_{N}, C_{T}$ at small values $\left(a=10 \mathrm{~mm}\right.$ and $n_{c}=100, C_{N_{-} M T}^{c \|}=869.86, C_{T_{-} M T}^{c \| l}=434.43$, case (c)).

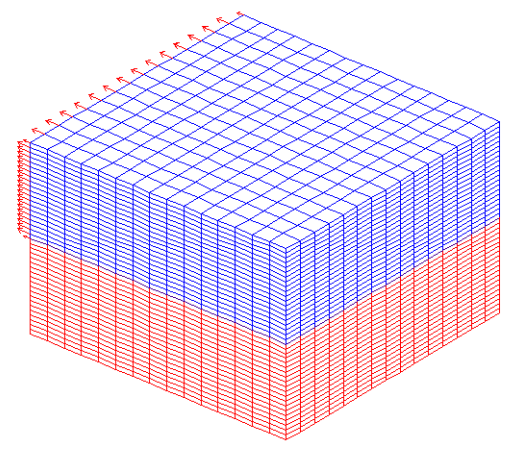

Figure 4. Force and finite element simulation in Cast3M. 


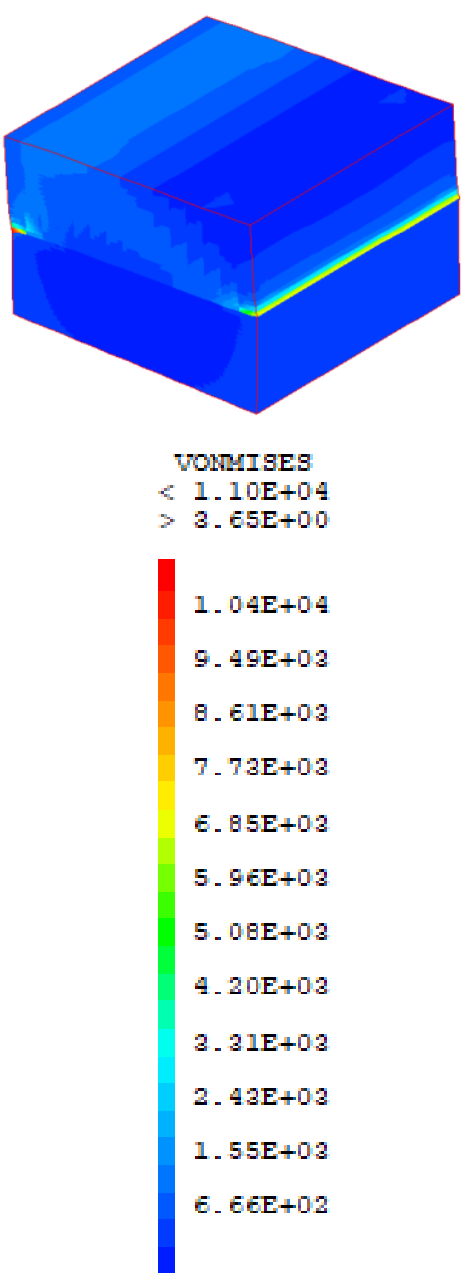

(a) Without interface
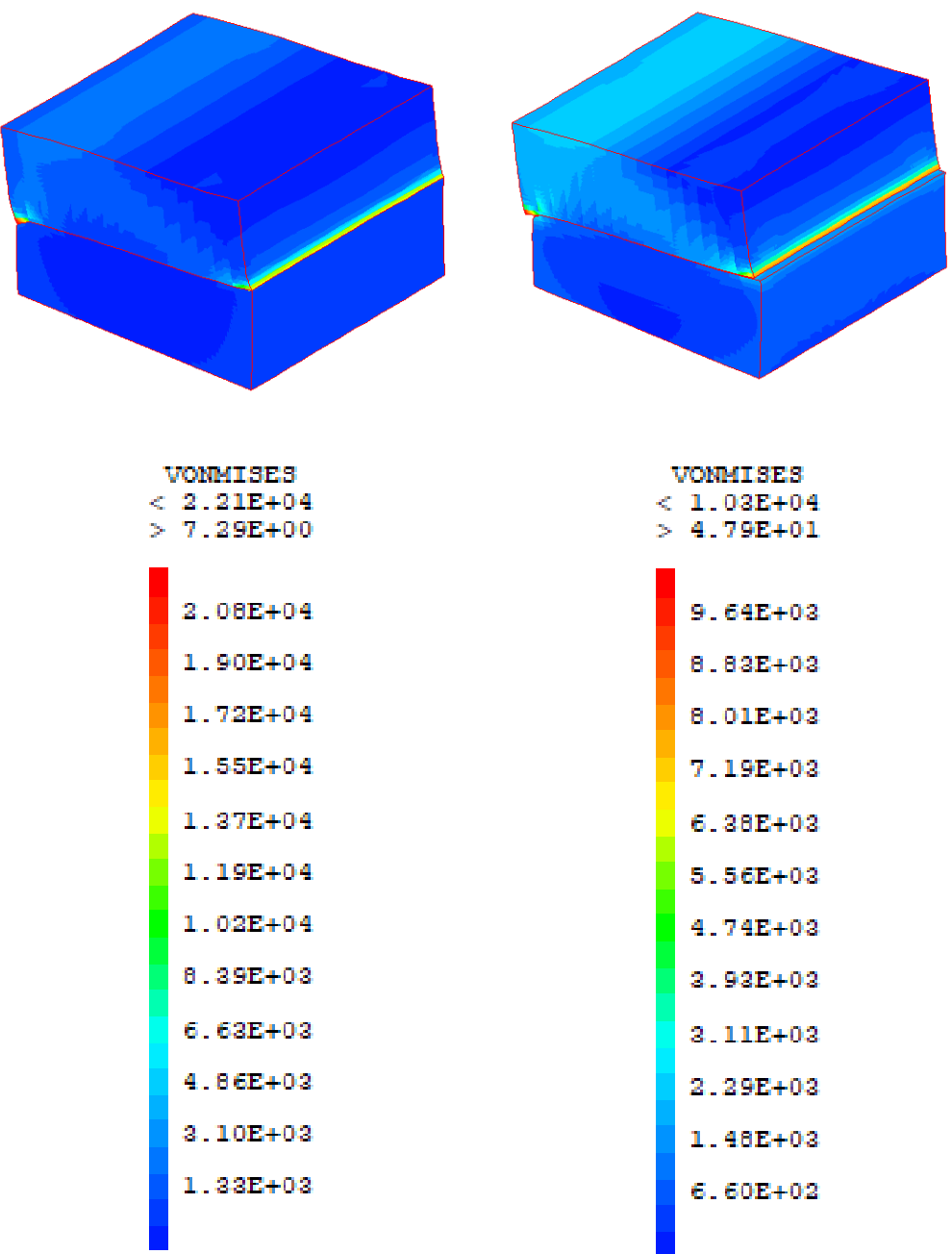

(b) With large stiffnesses

(c) With small stiffnesses

Figure 5. Stress distribution of hybrid structure.

Fig. 5 and Fig. 6 show that the concentration of stress occurs on two edges of the interface in all cases but case (a) does not cause sliding between the interfaces while the sliding is observed in case (b) and (c). The maximum displacement in case (a) is $4 \mathrm{~mm}$ in the middle zone of the interface, but the displacements at the edges are always equal to 0 (see Fig. 6). When $C_{N}, C_{T}$ are large, the displacement values at the interface are close to those of case (a) but one can observe a small slip in the opposite edge of the loading edge. Only case (c) with small stiffnesses gives overall slip (illustrated by the orange line in Fig. 6). Besides, when the interface sliding is clearly observed (case (c)), the stress concentration values in the two edges decrease (see Fig. 5). However, the stress distribution is more different between the two layers where the top layer occurs the larger stress. Therefore, the crack initiation point in the edges may occur at ultimate horizontal load before the sudden failure in the interface which breaks apart the layers. This result is suitable compared with the experimental test results in the literature [14-16]. 


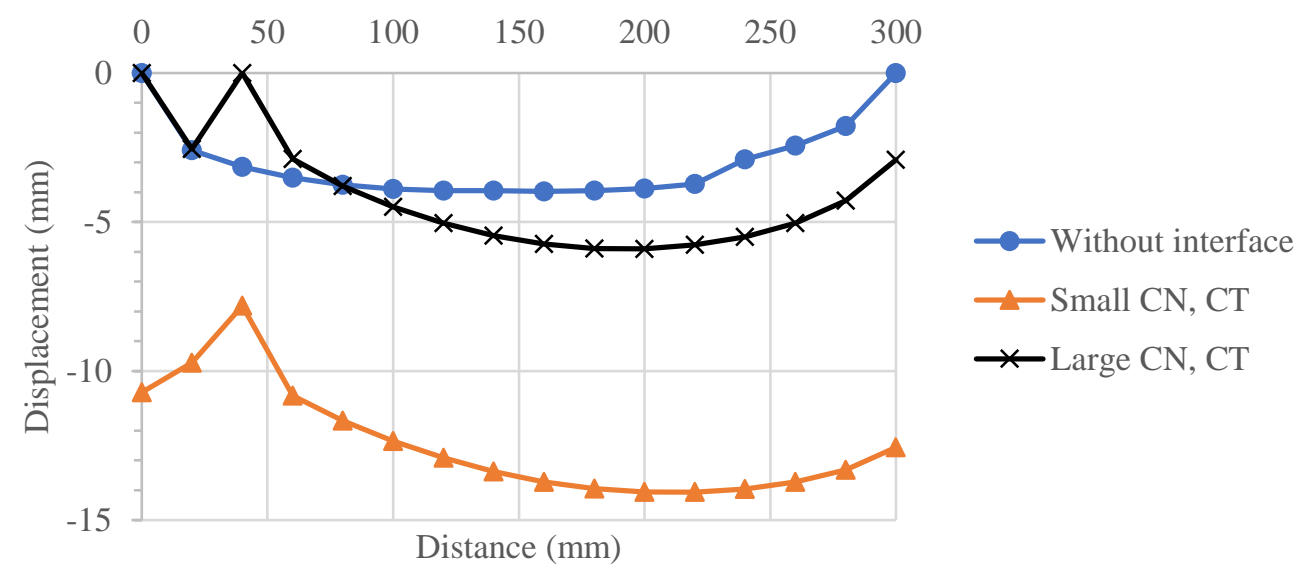

Figure 6. Medium displacement Ux along the interface.

\section{CONCLUSIONS}

In this study, a new method determining the stiffnesses of the interface is proposed for hybrid structures containing material layers with different mechanical properties and thickness based on the coupling between homogenization technique and asymptotic analysis.

The proposed method shows more advantages than the existing methods. Concretely, the stiffnesses are easily determined through the number and length of cracks and the dry or saturated states of the medium is considered. The results obtained are in accordance with the results obtained from the experimental studies.

Extension of this work concerning the validation of the proposed model with propagation of crack is considered as a perspective. With this aim, the hybrid structures will be considered until the maximum load reaches in which $C_{N}, C_{T}$ being functions of crack density and time.

\section{ACKNOWLEDGMENT}

This research is funded by Vietnam National Foundation for Science and Technology Development (NAFOSTED) under grant number 107.01-2017.307.

\section{REFERENCES}

[1]. Djamai, Zakaria Ilyes, et al., Textile reinforced concrete multiscale mechanical modelling: Application to TRC sandwich panels, Finite Elements in Analysis and Design, 135 (2017) 22-35. https://doi.org/10.1016/j.finel.2017.07.003

[2]. Lesley H. Sneed, Tommaso D'Antino, Christian Carloni, Investigation of bond behavior of PBO fiber-reinforced cementitious matrix composite-concrete interface, ACI Mater J., 111 (2014) 569580. https://doi.org/10.14359/51686604

[3]. Tetta Zoi C., Lampros N. Koutas, Dionysios A. Bournas, Textile-reinforced mortar (TRM) versus fiber-reinforced polymers (FRP) in shear strengthening of concrete beams, Composites Part B: Engineering, 77 (2015) 338-348. https://doi.org/10.1016/j.compositesb.2015.03.055

[4]. Cocu Marius, Existence of solutions of Signorini problems with friction, International journal of 
Transport and Communications Science Journal, Vol. 71, Issue 4 (05/2020), 419-430

engineering science, 22 (1984) 567-575. https://doi.org/10.1016/0020-7225(84)90058-2

[5]. Chetouane Brahim, et al., NSCD discrete element method for modelling masonry structures, International journal for numerical methods in engineering, 64 (2005) 65-94. https://doi.org/10.1002/nme.1358

[6]. V.Acary, Contribution à la modélisation mécanique et numérique des édifices maçonnés, 2001. (Doctoral dissertation).

https://www.researchgate.net/publication/30511955_Contribution_a_la_modelisation_mecanique _et_numerique_des_edifices_maconnes

[7]. Rekik Amna, Frédéric Lebon, Homogenization methods for interface modeling in damaged masonry, Advances in Engineering Software, $46 \quad$ (2012) 35-42. https://doi.org/10.1016/j.advengsoft.2010.09.009

[8]. Nguyen Thi Thu Nga, Interface elements for numerical simulation of masonry structures, Tap chi Xay dung, 7 (2017) 112-115 (in Vietnamese: Sử dụng phần tử tiếp xúc trong mô phỏng số kết cấu khối xây).

https://www.researchgate.net/publication/340526115_Su_dung_phan_tu_tiep_xuc_trong_mo_phong _so_ket_cau_khoi_xay_Interface_elements_for_numerical_simulation_of_masonry_structures

[9]. Benveniste Yakov, A new approach to the application of Mori-Tanaka's theory in composite materials, Mechanics of materials, 6 (1987) 147-157. https://doi.org/10.1016/0167-6636(87)90005-6

[10]. Thomsen Leon, Elastic anisotropy due to aligned cracks in porous rock, Geophysical Prospecting, 43 (1995) 805-829. https://doi.org/10.1111/j.1365-2478.1995.tb00282.x

[11]. Brown Robert JS, Jan Korringa, On the dependence of the elastic properties of a porous rock on the compressibility of the pore fluid, Geophysics, 40 (1975) 608-616. https://doi.org/10.1190/1.1440551

[12]. B. Shafiro, M. Kachanov, Materials with fluid-filled pores of various shapes: effective elastic properties and fluid pressure polarization, International Journal of Solids and Structures, 34 (1997) 3517-3540. https://doi.org/10.1016/S0020-7683(96)00185-0

[13]. T. T. N., Nguyen, Approches multi-échelles pour des maçonneries visco-élastiques, PhD thesis, France, 2015. http://www.theses.fr/2015ORLE2077

[14]. X. Z. Lu et al., Finite element simulation of debonding in FRP-to-concrete bonded joints, $\begin{array}{lllll}\text { Construction and building materials, } 20 \quad \text { (2006) } & \end{array}$ https://doi.org/10.1016/j.conbuildmat.2005.01.033

[15]. Abdulla Kurdo F., Lee S. Cunningham, Martin Gillie, Simulating masonry wall behaviour using a simplified micro-model approach, Engineering Structures, 151 (2017) 349-365. https://doi.org/10.1016/j.engstruct.2017.08.021

[16]. Mang Chetra, Ludovic Jason, Luc Davenne, A new bond slip model for reinforced concrete structures, Engineering Computations, 32 (2015) 1934-1958. https://doi.org/10.1108/EC-11-20140234

[17]. Mindess Sidney, Sidney Diamond, The cracking and fracture of mortar, Matériaux et Construction, 15 (1982) 107-113. https://doi.org/10.1007/BF02473571 